\title{
Elaboração e implantação do sistema de análise de perigos e pontos críticos de controle no processamento de leite pasteurizado tipo $\mathrm{A}$
}

\author{
Development and implementation of the hazard analysis and critical control points \\ system for the processing of grade A pasteurized milk
}

Wanderleia Tobias $^{\mathrm{I}}$ Elisa Helena Giglio Ponsano ${ }^{\mathrm{I}}$ Marcos Franke Pinto $^{\mathrm{I}}$

RESUMO

A análise de perigos e pontos críticos de controle (APPCC) é uma das principais ferramentas utilizadas atualmente para a garantia da inocuidade, qualidade e integridade dos alimentos. $O$ objetivo deste trabalho foi elaborar e implantar o sistema APPCC no processamento do leite tipo A pasteurizado. Por meio da aplicação de listas de verificação, foi avaliado o nível do programa de pré-requisitos e o panorama sanitário do estabelecimento, e o material gerado serviu de suporte para a implantação do sistema. O modelo de "árvore decisória” foi utilizado para a determinação dos Pontos Críticos de Controle (PCC), tendo sido identificados como PCC biológicos as etapas de pasteurização e envase e nenhum PCC químico ou físico. Para os PCC identificados, foram estabelecidas as medidas preventivas $e$ corretivas, os limites críticos e os procedimentos de monitoramento e verificação. O programa de pré-requisitos foi fundamental para o estabelecimento do sistema. A implantação do plano APPCC no processamento do leite pasteurizado tipo A foi eficiente no controle dos perigos identificados e possibilitou ao produto atingir as especificações legais e alcançar a segurança.

Palavras-chave: tecnologia de alimentos, segurança alimentar sanitária, manipulação de alimentos, legislação sobre alimentos.

\section{ABSTRACT}

Hazard analysis and critical control points (HACCP) is one of the main tools currently used to ensure safety, quality and integrity of foods. So, the aim of this study was to develop and implement the HACCP program in the processing of pasteurized grade A milk. Checklists were used to assess on the level of the pre requisites programs and on the sanitary classification of the dairy industry and the results were used as references for the development of the HACCP system. A "decision tree" protocol was used for the identification of the critical control points (CCP). No physical or chemical CCP were identified, whereas pasteurization and packaging were considered biological CCP. For these CCP, the limits for prevention, monitoring needs, corrective actions, critical limits and verification procedures were established. The pre requisites program was essential for the establishment of the system. The implementation of the HACCP for the processing of grade A pasteurized milk was efficient to control the biological hazards and enabled the product to comply with the legislation specifications and achieve safety.

Key words: food technology, food safety, food handling, food legislation.

\section{INTRODUÇÃO}

A principal ferramenta utilizada na atualidade para garantir inocuidade, qualidade e integridade dos alimentos é a Análise de Perigos e Pontos Críticos de Controle (APPCC) (KHATRY \& COLLINS, 2007; VIOLARIS et al., 2008), um sistema que abrange a prevenção dos perigos associados com a produção ou uso dos alimentos e a identificação de pontos que permitem o controle desses perigos (FORSYTHE, 2002).

Em 1998, o Ministério da Agricultura, Pecuária e Abastecimento (MAPA) instituiu a aplicação do sistema APPCC nas indústrias de produtos de origem animal em nível nacional (BRASIL, 1998) e, a partir de 2001, iniciou auditorias nas indústrias de laticínios do Brasil, fazendo com que esses estabelecimentos passassem a empenhar-se na implantação do sistema (BRUM, 2004). O sistema APPCC estabelecido pelo MAPA segue o modelo proposto pelo Codex Alimentarius e contempla

'Faculdade de Medicina Veterinária, Universidade Estadual Paulista (Unesp), Rua Clóvis Pestana, 793, 16050-680, Araçatuba, SP, Brasil. E-mail: elisahgp@fmva.unesp.br.*Autor para correspondência. 
sete princípios básicos, que são: Análise de perigos; Determinação dos Pontos Críticos de Controle (PCC); Estabelecimento do(s) limite(s) crítico(s); Estabelecimento de ações de monitoramento dos PCC; Estabelecimento de ações corretivas para o controle dos PCC; Estabelecimento de procedimentos para a verificação do funcionamento do sistema; e Estabelecimento de um sistema de documentação e registro(CODEXALIMENTARIUS COMMISSION, 2003).

O sucesso e a efetividade do sistema APPCC na prevenção de doenças de origem alimentar e de riscos à saúde do consumidor dependem da correta aplicação desses princípios, combinados com os programas de pré-requisitos, que incluem as Boas Práticas de Fabricação (BPF), os Procedimentos Operacionais Padronizados (POP) e os Procedimentos Padrão de Higiene Operacional (PPOH) (BRASIL, 1998; ROBERTO et al., 2006). As BPF contêm requerimentos detalhados para evitar a ocorrência de contratempos durante o processo produtivo e, por isso, são uma parte indispensável de todo sistema de qualidade (SANDROU \& ARVANITOYANNIS, 2000). Em 1997, a utilização das BPF como instrumento de fiscalização passou a ser regulamentada pelo Ministério da Saúde MS (BRASIL, 1997b) e pelo Ministério da Agricultura e do Abastecimento - MAA (BRASIL, 1997a). Os PPHO preconizados pelo órgão americano Food and Drug Administration (FDA) constituíram, até outubro de 2002, a referência para o controle de procedimentos de higiene no Brasil. A partir daí, a Agência Nacional de Vigilância Sanitária (ANVISA) estabeleceu os POP, que vão um pouco além do simples controle de higiene e são definidos como procedimentos escritos de forma objetiva, que estabelecem instruções sequenciais para a realização de operações rotineiras e específicas na produção, armazenamento e transporte de alimentos (BRASIL, 2002).

Diante desse quadro, o Sistema APPCC, associado às BPF e aos POP, tem se revelado como ferramenta básica do sistema moderno de gestão da qualidade nas indústrias de alimentos, garantindo a segurança dos produtos, reduzindo custos e aumentando a lucratividade, por meio da diminuição das perdas e do retrabalho. Além disso, essas ferramentas da qualidade aperfeiçoam processos e tornam desnecessárias boa parte das análises laboratoriais realizadas no sistema de controle de qualidade tradicional, tornando o processo de controle transparente e confiável (CNI, 2002; SUN \& OCKERMAM, 2005).

Apesar de apresentar um inestimável valor nutricional a um custo acessível, o leite está sujeito a sofrer contaminações com agentes de ordem química (resíduos de produtos de limpeza, sanitizantes, medicamentos, etc.), física (pelos, fragmentos metálicos, etc.) ou biológica (microrganismos patogênicos), em decorrência de falhas higiênicas, sanitárias e operacionais na cadeia produtiva, tornando-se um potencial veículo de doenças de origem alimentar. Com o intuito de garantir o consumo seguro do leite oferecido à população regional e de atender à regulamentação vigente, o objetivo deste trabalho foi elaborar e implantar o plano APPCC no processamento do leite pasteurizado tipo A.

\section{MATERIAL E MÉTODOS}

As etapas para a elaboração e a implantação do plano APPCC foram desenvolvidas de acordo com o preconizado em BRASIL (1998), sendo elas:

- Formação da equipe responsável pela elaboração e implantação do plano APPCC, incluindo um supervisor, um coordenador e os executores;

- Identificação da empresa;

- Avaliação dos pré-requisitos (BPF e POP) e classificação do estabelecimento quanto ao panorama sanitário, realizadas a partir da aplicação de lista de verificação, abrangendo os tópicos: "Edificações e instalações”; "Equipamentos móveis e utensílios”; "Manipuladores"; "Produção e transporte do alimento" e "Documentação" (BRASIL, 2002). O diagnóstico inicial foi realizado em abril de 2011 e o diagnóstico final em abril de 2012, utilizando-se, em ambos, a mesma lista de verificação, a fim de se avaliar a evolução da implantação das BPF e do panorama sanitário. Os POP foram desenvolvidos e implantados segundo BRASIL (2002).

- Capacitação técnica dos funcionários;

- Descrição do produto e seu uso pretendido;

- Elaboração do diagrama operacional do processamento do leite tipo A pasteurizado;

- Sequência lógica de aplicação dos sete princípios básicos do APPCC, em que foi utilizado o modelo de “árvore decisória” para orientar a identificação dos PCC. Para o monitoramento das etapas consideradas PCC, foram realizadas a aferição do binômio tempo x temperatura (na pasteurização) e a inspeção visual (no envase). Para a verificação do sistema APPCC, foram utilizadas as provas da fosfatase e a Contagem Padrão em Placas (CPP) no leite (BRASIL, 2011) e a análise microbiológica da superfície das embalagens (SILVA, 2007).

- Encaminhamento da documentação para avaliação pela Coordenadoria da Defesa Agropecuária do Estado de São Paulo. 
- Aprovação, implantação e validação do plano APPCC.

\section{RESULTADOS E DISCUSSÃO}

$\mathrm{Na}$ auditoria inicial, foram avaliados 172 itens, sendo 67 considerados conformes e 95 considerados não-conformes. Para dez itens, não houve aplicação e, assim, o percentual de conformidade alcançado foi de $38,95 \%$, classificando o estabelecimento no grupo 3 do panorama sanitário (Grupo 1-76 a 100\% dos itens atendidos; Grupo 2-51 a $75 \%$ dos itens atendidos; Grupo $3-0$ a 50\% dos itens atendidos). Os itens "Manipuladores", "Edificações e instalações" e "Documentação" foram os que apresentaram maiores índices de não conformidade, $71,43 \%, 57,14 \%$ e $100 \%$, respectivamente. Em relação aos manipuladores, as não conformidades deveram-se à falta de treinamento em relação à higiene e conduta pessoal; em relação às instalações, os principais pontos de não conformidade deveram-se à falta de isolamento e pavimentação na entrada da empresa e à falta de manutenção de paredes e telas das janelas da área interna, além da inexistência de um programa adequado para o controle de pragas e, quanto à documentação, verificou-se que não havia registro das atividades envolvidas na produção e na industrialização do leite na empresa. BRUM (2004) aplicou uma lista similar à utilizada neste trabalho para a verificação das BPF no processamento de leite pasteurizado e encontrou não conformidades em 35\% dos itens avaliados, sendo a maioria delas devidas ao não cumprimento dos mesmos itens detectados neste trabalho.

Com o resultado da primeira auditoria, constatou-se o baixo nível de aderência da empresa às BPF e a necessidade de correção das não conformidades. Assim, foram realizadas atividades de capacitação dos colaboradores, tais como palestras e treinamento prático no atendimento às $B P F$, adequação dos pontos físicos não conformes, escolha e adoção de um programa de controle de pragas e elaboração da documentação referente às BPF. Nesse momento, o empenho da diretoria foi essencial para a implantação do APPCC, uma vez que essas correções envolveram investimentos extras. OLIVEIRA et al. (2013) ressaltam que a diminuição na velocidade da realização de ações corretivas que envolvem investimentos diminui a eficácia da implantação do plano. No entanto, de acordo com ROBERTO et al. (2006), a longo prazo, o retorno do investimento é verificado pela redução de produtos contaminados, pela melhoria da qualidade e segurança do alimento, pelo aumento na confiabilidade e pela diminuição de reclamações.

Na auditoria final, dos 172 itens avaliados, 157 foram considerados conformes e cinco não conformes, o que proporcionou um aumento para $91,27 \%$ de itens atendidos e permitiu ao estabelecimento alcançar o grupo 1 do panorama sanitário. A maior parte das não conformidades ainda encontradas após a auditoria final referiram-se ao item "Edificações e instalações", e suas correções ficaram a cargo da diretoria da empresa. O manual de BPF contemplando os itens necessários segundo a legislação vigente foi elaborado e implantado e também foram elaborados os POP, contemplando os itens: higienização das instalações, equipamentos e móveis; higiene e conduta pessoal; controle de pragas; potabilidade da água; manutenção dos equipamentos/ calibração; e destinação de resíduos. De acordo com OLIVEIRA \& MASSON (2003), a implantação das BPF representa um passo importante para o APPC, já que determinados pontos críticos podem ser controlados pelo programa de pré-requisitos. Ainda, de acordo com os autores, quando isso não ocorre, acaba por sobrecarregar o sistema, ficando a sua eficiência comprometida pela necessidade de administração de muitos controles.

Com a elaboração do organograma da empresa, ficou definida a equipe do APPCC, com as atribuições e responsabilidades de cada participante: o gerente como supervisor, o médico veterinário como coordenador e os colaboradores como executores do sistema. Os integrantes da equipe APPCC devem ser pessoas com poder de convencimento e liderança e capacidade de multiplicação dos conhecimentos obtidos, de modo a possibilitar a penetração dos conceitos do programa nos diversos setores do estabelecimento industrial e a facilitar a sensibilização do corpo funcional para a importância do plano (BRASIL, 1998).

A análise da criticidade realizada para a matéria prima não identificou perigos críticos de qualquer natureza, uma vez que o processamento é capaz de eliminá-los ou reduzi-los a níveis aceitáveis, caso estejam presentes. O fluxograma de processamento do leite, contemplando os PCC, está apresentado na figura 1. Para BRITO et al. (2004) e ROSA \& QUEIROZ (2007), a elaboração do fluxograma fornece detalhes úteis para a implantação do APPCC e permite a identificação de problemas potenciais, tal como a realização da homogeneização posteriormente à pasteurização (SANDROU \& ARVANITOYANNIS, 2000). Os fundamentos das etapas tecnológicas envolvidas no processamento 


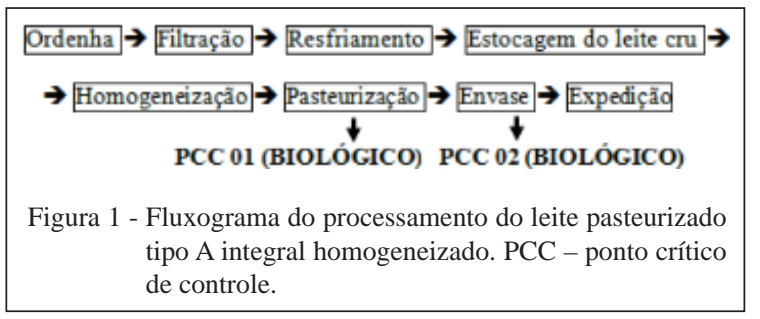

estão descritos na tabela 1 e a descrição e a indicação de uso do produto são apresentados na tabela 2.

Segundo BRASIL (1998), todos os perigos identificados, associados a cada etapa do processo, devem ser listados e sua severidade avaliada. A equipe responsável pelo APPCC realizou a análise dos riscos e, como resultado, foram identificados como PCC as etapas de pasteurização e envase, conforme o resumo do plano apresentado na tabela 3.
Em seu trabalho de implantação de programas de qualidade na produção de leite pasteurizado tipo C, BRUM (2004) identificou a etapa de ordenha como sendo um PCC, devido à presença de perigos químicos e biológicos. Naquele caso, porém, a matéria prima era recebida de fornecedores, diferente do que ocorre na produção de leite pasteurizado tipo A, em que a matéria prima é produzida e processada na propriedade e esses perigos são controlados pelas boas práticas na ordenha. Portanto, neste trabalho, essa etapa não foi considerada um PCC. VARNAM \& SUTHERLAND (1994) consideram a etapa de homogeneização no processamento de leite pasteurizado como um PCC biológico quando essa operação é realizada após a pasteurização, o que não permite a adoção de nenhuma medida de controle para garantir a segurança do produto. Neste trabalho, porém, essa etapa foi realizada antes da pasteurização

Tabela 1 - Descritivo do processo de fabricação de leite pasteurizado tipo A integral homogeneizado.

Matéria prima

Resfriamento e inspeção da qualidade da matéria prima

Homogeneização

Pasteurização

Envase e inspeção da qualidade do produto final

Expedição
Todo o leite utilizado na granja leiteira é proveniente de rebanho próprio, mantido sob controle sanitário, com assistência diária de médico veterinário. A ordenha e a filtração são realizadas por meio mecânico em sistema fechado, em instalações apropriadas que obedecem aos requisitos de construção e funcionamento preconizados pela legislação. Os procedimentos higiênico-sanitários preconizados para a obtenção do leite são rigorosamente respeitados (BRASIL, 2011).

A temperatura do leite na ocasião da ordenha $\left( \pm 35^{\circ} \mathrm{C}\right)$ é bastante favorável à multiplicação dos microrganismos e o resfriamento funciona como uma medida eficaz para conter a acidificação. O processo é realizado por meio do bombeamento automático do leite em circuito fechado desde a sala de ordenha, passando por sistema de placas de resfriamento até chegar ao tanque regulador. Nesse ponto, uma amostra do leite é analisada quanto a: Contagem Padrão em Placas (CPP); Contagem de Células Somáticas (CCS); pesquisa de resíduos de antibióticos; índice crioscópico; teor de sólidos totais e não-gordurosos; teor de gordura; densidade relativa; acidez titulável; estabilidade ao alizarol $72 \%$ (v/v) e medição da temperatura (BRASIL, 2011)

O processo ocorre por meio mecânico em homogeneizador, a fim de diminuir o tamanho das partículas de gordura, com o objetivo de evitar a formação de camada de creme na superfície do leite empacotado durante o período de validade. O leite é forçado em alta velocidade através da passagem por um orifício pequeno, gerando alta pressão que, em combinação com a turbulência, provoca a ruptura dos glóbulos de gordura em porções menores, de menor tendência à aglomeração.

Consiste no aquecimento do leite entre 72 a $75^{\circ} \mathrm{C}$, durante 15 a 20 segundos, seguido de resfriamento para temperatura inferior a $4^{\circ} \mathrm{C}$, em equipamento de pasteurização a placas, dotado de painel de controle com termorregistrador, termorregulador e válvula de desvio de fluxo automáticos, termômetros e torneiras de prova. Essa etapa visa eliminar organismos patogênicos e reduzir os deterioradores, aumentando a vida útil do leite, sem promover alteração sensível de sua composição nutricional e sensorial.

Ao final do processamento, o leite é envasado em embalagens plásticas devidamente rotuladas e imediatamente armazenado em câmara fria. A embalagem recebe um tratamento com luz UV previamente a seu enchimento e soldagem, dentro do próprio equipamento. Amostras aleatórias são coletadas da câmara fria e analisadas (no primeiro e no último dia de validade do produto) por meio das seguintes provas (BRASIL, 2011): CPP; Coliformes a $30 / 35^{\circ} \mathrm{C}$; Coliformes a $45^{\circ} \mathrm{C}$; Salmonella spp; teor de gordura; acidez titulável; estabilidade ao alizarol $72 \%(\mathrm{v} / \mathrm{v})$; sólidos não gordurosos; índice crioscópico; provas de fosfatase alcalina e peroxidase. 
Tabela 2 - Descrição e uso pretendido do leite pasteurizado tipo A integral homogeneizado

Nome do produto-

LEITE PASTEURIZADO TIPO A INTEGRAL HOMOGENEIZADO

\begin{tabular}{|c|c|}
\hline Ingrediente & Leite in natura \\
\hline \multirow{4}{*}{ Características físicas e químicas do produto final } & $a_{w}: 0,83$ a 0,86 \\
\hline & $\mathrm{pH}: 6,4$ a 6,8 \\
\hline & Gordura (g/100g): mínimo 3\% \\
\hline & Proteína total (g/100g): mínimo 2, 9\% \\
\hline \multirow{8}{*}{ Características microbiológicas do produto final } & Contagem Padrão em Placas (UFC mL $L^{-1}$ ) \\
\hline & $\mathrm{n}=5 ; \mathrm{c}=2 ; \mathrm{m}=5 \times 10^{2} ; \mathrm{M}=1 \times 10^{3}$ \\
\hline & Coliformes NPM mL $\mathrm{m}^{-1}\left(30 / 35^{\circ} \mathrm{C}\right)$ \\
\hline & $\mathrm{n}=5 ; \mathrm{c}=0 ; \mathrm{m}<1$ \\
\hline & Coliformes NPM mL $\mathrm{mL}^{-1}\left(45^{\circ} \mathrm{C}\right)$ \\
\hline & $\mathrm{n}=5 ; \mathrm{c}=0 ; \mathrm{m}=$ ausência \\
\hline & Salmonella spp $25 \mathrm{~mL}^{-1}$ \\
\hline & n=5; c=0; m=ausência \\
\hline Validade & 4 dias após a fabricação \\
\hline Informações para o consumidor & Não contém glúten \\
\hline \multirow{2}{*}{ Informações para armazenagem, transporte e distribuição } & Manter resfriado de 1 a $4^{\circ} \mathrm{C}$ \\
\hline & Após aberto, consumir em 4 dias \\
\hline Locais de comercialização & Distribuidores atacadistas, supermercados \\
\hline Uso pretendido & Comercialização \\
\hline Embalagem & Primária: filme de poliéster \\
\hline
\end{tabular}

$\mathrm{a}_{\mathrm{w}}$ - atividade de água; U.F.C. - Unidades Formadoras de Colônias; NMP- Número Mais Provável; n- número de unidades retiradas de um lote que serão analisadas independentemente (unidades amostrais); c - número máximo aceitável de unidades do lote em que as contagens microbianas estão acima do limite mínimo (m) e abaixo do limite máximo (M) tolerado para o microrganismo investigado; m -limite mínimo; M - limite máximo.

e, assim, não foi considerada um PCC. A classificação da etapa de pasteurização como um PCC biológico se justifica pelo fato de que, nesse ponto, caso haja uma falha no binômio tempo x temperatura, poderá haver a sobrevivência de microrganismos patogênicos (VARNAM \& SUTHERLAND, 1994). Quanto à etapa de envase, ela foi considerada um PCC biológico porque, quando realizada de forma correta, irá assegurar a qualidade microbiológica nas etapas seguintes. Pelos mesmos motivos, VALADÃO (2012) também considerou a pasteurização e o envase como PCC em seu estudo com leite tipo A.

Para o PCC pasteurização, a medida preventiva adotada foi a manutenção do binômio tempo $\mathrm{x}$ temperatura dentro do estipulado pela legislação (72 a $75^{\circ} \mathrm{C} / 15$ a 20 segundos) (BRASIL, 2011) e, como limite crítico, adotou-se o binômio $75^{\circ} \mathrm{C}$ por 15 segundos. Ficou estabelecido que o monitoramento dessa etapa seja realizado pelos colaboradores, fazendo-se o registro da temperatura no pasteurizador. As medidas de controle adotadas foram o cumprimento dos procedimentos de monitoramento do equipamento de pasteurização, assim como a execução de manutenção corretiva e preventiva, de acordo com as BPF. Ficou estabelecido que, diariamente, sejam realizados testes com o objetivo de verificar o correto funcionamento da válvula de fluxo, que deve promover o desvio do leite que eventualmente não tenha atingido o aquecimento requerido pelo processo de pasteurização, e que sejam seguidas as recomendações dos fabricantes quanto ao tempo máximo de operação dos equipamentos e aos procedimentos de limpeza e sanitização. Ainda ficou estabelecido que, ocorrendo o desvio do limite crítico, a medida corretiva a ser realizada seja a segregação do produto e que, após a realização da análise de fosfatase, caso o limite crítico tenha sido perdido, o leite deverá ser reprocessado, sendo efetuado o registro do procedimento realizado.

Para o envase, as medidas preventivas adotadas incluíram a correta execução diária da sanitização da máquina envasadora e a limpeza e 
Tabela 3 - Resumo do plano de Análise de Perigos e Pontos Críticos de Controle para a produção de leite pasteurizado tipo A integral homogeneizado.

\begin{tabular}{|c|c|c|}
\hline & Pasteurização & Envase \\
\hline PCC & PCC 01 (Biológico) & PCC 02 (Biológico) \\
\hline Perigo & $\begin{array}{l}\text { Sobrevivência de taxas elevadas de microrganismos } \\
\text { patogênicos }\end{array}$ & Contaminação microbiana \\
\hline $\begin{array}{l}\text { Medidas } \\
\text { preventivas }\end{array}$ & Adoção de binômio tempo x temperatura adequados & $\begin{array}{l}\text { Seleção de fornecedores, BPF, manutenção adequada da } \\
\text { máquina de envase e das lâmpadas UV }\end{array}$ \\
\hline Limite crítico & $75^{\circ} \mathrm{C} 15 \mathrm{~s}$ & Lâmpada UV em funcionamento \\
\hline \multirow{4}{*}{ Monitoramento } & O quê? Temperatura & O quê? Lâmpadas UV \\
\hline & Como? Verificação do termômetro & Como? Inspeção visual \\
\hline & Quando? A cada pasteurização & Quando? No início e durante o processo \\
\hline & Quem? Executor do APPCC & Quem? Executor do APPCC \\
\hline Ação corretiva & Segregar, ajustar tempo e temperatura, reprocessar & $\begin{array}{l}\text { Segregar, ajustar a máquina, refazer a higienização, } \\
\text { reprocessar }\end{array}$ \\
\hline Registros & $\begin{array}{l}\text { Planilhas de tempo e temperatura, laudo de teste da } \\
\text { fosfatase }\end{array}$ & Planilhas de programa de limpeza (BPF) \\
\hline Verificação & $\begin{array}{l}\text { Teste da fosfatase, Contagem Padrão em Placas e } \\
\text { auditorias internas }\end{array}$ & $\begin{array}{l}\text { Análise microbiológica da superfície da embalagem e } \\
\text { monitoramento das BPF }\end{array}$ \\
\hline
\end{tabular}

PCC - Ponto Crítico de Controle; BPF - Boas Práticas de Fabricação.

manutenção da lâmpada de esterilização, por meio da aplicação das BPF. Além disso, ficou estabelecido que seja realizada uma rigorosa seleção dos fornecedores de embalagens, que deverão ter qualidade microbiológica atestada por laudos técnicos e por programas de boas práticas. Como limite crítico, adotou-se o funcionamento das lâmpadas UV, que deverá ser monitorado visualmente antes e durante o processo. Ficou estabelecido que, caso seja detectado o mau funcionamento da lâmpada no monitoramento, o leite deverá ser reprocessado e o procedimento registrado.

Neste trabalho, foi possível observar a importância das BPF para o controle dos perigos identificados. Ao contrário, BRUM (2004) concluiu que a aplicação do APPCC na produção de leite pasteurizado tipo $\mathrm{C}$ não foi satisfatória, devido à não implantação das BPF, o que ressalta a importância dos programas de pré-requisitos para a implantação desse sistema.

A Contagem Padrão em Placas, realizada mensalmente na verificação do sistema APPCC, indicou valores abaixo do limite máximo de $1 \times 10^{3} \mathrm{UFC} \mathrm{mL}^{-1}$, estabelecido pela Instrução Normativa oㅡ 62 (BRASIL, 2011), representando uma redução de $20 \%$ nos valores encontrados antes da implantação, e a pesquisa da fosfatase alcalina apresentou sempre resultados negativos, colocando o produto dentro das especificações legais. As análises de superfície das embalagens apresentaram sempre contagens de mesófilos aeróbios inferiores a 1UFC $\mathrm{cm}^{-2}$. Esses resultados permitem inferir que, uma vez atendidos os preceitos legais, a vida de prateleira do produto estará garantida, assim como estará alcançada a segurança do alimento. O mesmo não ocorreu no trabalho de VALADÃO (2012), em que os resultados das análises realizadas na verificação dos PCC demonstraram que os estabelecimentos produtores de leite tipo A estavam produzindo leite fora dos limites estabelecidos pela legislação.

Após a elaboração e a aplicação do plano APPCC, ele foi encaminhado à Coordenadoria de Defesa Agropecuária do Estado de São Paulo para ser validado. O plano deverá passar por processo de verificação de responsabilidade do governo como órgão regulador e das suas ações para garantir que 0 sistema esteja funcionado adequadamente

\section{CONCLUSÃO}

A implantação do plano APPCC no processamento do leite pasteurizado tipo A foi eficiente no controle dos perigos biológicos identificados, permitindo ao estabelecimento a adequação à legislação brasileira e garantindo a segurança do produto oferecido à população.

\section{REFERÊNCIAS}

BRASIL. Instrução Normativa n.62 de 29 de dezembro de 2011. Aprova o regulamento técnico de produção, identidade e qualidade do leite tipo $\mathrm{A}$, o regulamento técnico de identidade e qualidade de 
leite cru refrigerado e do leite pasteurizado e o regulamento técnico da coleta de leite cru refrigerado e seu transporte a granel. Diário Oficial [da] República Federativa do Brasil. Brasília, DF. 1 de janeiro de 2012.

BRASIL. Ministério da Agricultura e Abastecimento. Portaria n.46, de 10 de fevereiro de 1998. Institui o sistema de análise de perigos e pontos críticos de controle APPCC a ser implantado nas indústrias de produtos de origem animal. Diário Oficial [da] Republica Federativa do Brasil, Brasília, DF, 10 fev. 1998. Seção I.

BRASIL. Ministério da Agricultura e Abastecimento. Portaria n.368, de 04 de setembro de 1997. Aprova o Regulamento Técnico sobre as condições Higiênico-Sanitárias e de Boas Práticas de Fabricação para Estabelecimentos Elaboradores / Industrializadores de Alimentos. Diário Oficial [da] República Federativa do Brasil. Brasília, DF, 8 de setembro de 1997a.

BRASIL. Ministério da Saúde. Agência Nacional de Vigilância Sanitária(ANVISA). Resolução RDC n.275, de 21 de outubro de 2002. Regulamento Técnico de Procedimentos Operacionais Padronizados aplicados aos Estabelecimentos Produtores/Industrializadores de Alimentos e Lista de Verificação das Boas Práticas de Fabricação em Estabelecimentos Produtores/Industrializadores de Alimentos. Diário Oficial [da] República Federativa do Brasil. Brasília, DF, 23 outubro 2002. Seção 1, p. 126.

BRASIL. Ministério da Saúde. Portaria n.326, de 30 de julho de 1997. Aprova o Regulamento Técnico “Condições HigiênicoSanitárias e de Boas Práticas de Fabricação para Estabelecimentos Produtores/Industrializadores de Alimentos”. Diário Oficial [da] República Federativa do Brasil. Brasília, DF, 1 de agosto de 1997b.

BRITO, J.R.F. et al. Adoção de boas práticas agropecuárias em propriedades leiteiras da região Sudeste do Brasil como um passo para a produção de leite seguro. Acta Scientiae Veterinariae, v.32, n.2, p.125-131, 2004. Disponível em: <http://www.ufrgs.br/ actavet/32-2/artigo589.pdf>. Acesso em 21 out. 2013.

BRUM, J.V.F. Análise de perigos e pontos críticos de controle em indústrias de lacticínios de Curitiba- PR. 2004. 146f. Dissertação (Mestrado em Tecnologia de Alimentos) Universidade Federal do Paraná, Curitiba, PR.

CNI (CONFEDERAÇÃO NACIONAL DA INDÚSTRIA). Guia para implantação de boas práticas de fabricação. Brasília: SENAI/SEBRAE, 2002. 151p. Série Qualidade e Segurança Alimentar.

CODEX ALIMENTARIUS COMMISSION (CAC). Hazard analysis and critical control point (HACCP) system and guidelines for its application. ANNEX to recommended international code of practice/general principles of food hygiene. CAC/RCP 1-1969, Rev 4. Roma: FAO/WHO Codex Alimentarius Commission, 2003. 68 p.

FORSYTHE, S.J. Microbiologia da segurança alimentar. Porto Alegre: Artmed, 2002. 424 p.

KHATRY, Y; COLLINS, R. Impact and status of HACCP in the Australian meat industry. British Food Journal, v.109, p.343354, 2007. Disponível em: <www.emeraldinsight.com/0007-070X. htm>. Acesso em 20 out. 2013. doi: 10.1108/00070700710746768.
OLIVEIRA, A.M.; MASSON, M.L. Terminologia e definições utilizadas nos sistemas da qualidade e segurança alimentar. Boletim da SBCTA, v.37, n.1, p.52-57, 2003. Disponível em: <http://www.scielo. br/scielo.php?script=sci_arttext\&pid=S0101-20611997000400001>. Acesso em 15 abr. 2014.

OLIVEIRA, C.A.F. et al. Avaliação dos custos envolvidos na implementação de BPF e APPCC em uma indústria de laticínios de pequeno porte. Veterinária e Zootecnia, v.20, n.2, Supl.1, p.38-39, 2013. Disponível em: <http:file://C:/Users/usuario/ Downloads/707-2779-1-PB\%20(3).pdf>. Acesso em 24 jun. 2006.

ROBERTO, C.D. et al. Costs and investments of implementing and maintaining HACCP in a pasteurized milk plant. Food Control, v.17, p.599-603, 2006. Disponível em: <http://ac.elscdn.com/S0956713504001161/1-s2.0-S0956713504001161-main. pdf?_tid=a9107178-c4ad-11e3-a4a9-00000aacb361\&acdnat=139 7573769_05abf96cb46b9756748308898ea621f8>. Acesso em 15 abr. 2014. doi: 10.1016/j.foodcont.2004.05.011.

ROSA, L.S.; QUEIROZ, M.I. Avaliação da qualidade do leite cru e resfriado mediante a aplicação de princípios do APPCC. Ciência e Tecnologia de Alimentos, v.27, n.2, p.422-430, 2007. Disponível em: <http://www.scielo.br/pdf/cta/v27n2/35.pdf>. Acesso em 28 ago. 2008.

SANDROU, D.K.; ARVANITOYANNIS, I.S. Implementation of hazard analysis critical control point (HACCP) to the dairy industry: current status and perspectives. Food Reviews International, v.16, n.1, p.77-111, 2000. Disponível em: <http:// www.tandfonline.com/doi/pdf/10.1081/FRI-100100283>. Acesso em 22 out. 2013. doi: 10.1081/FRI-100100283.

SILVA, N. et al. Manual de métodos de análise microbiológica de alimentos. 3.ed. São Paulo: Varela, 2007. 544 p.

SUN, Y.M.; OCKERMAN, H.W. A review of needs and current applications of hazard analysis and critical control point (HACCP) in food service areas. Food Control, v.16, p.325-332, 2005. Disponível em: <http://ac.els-cdn.com/S0956713504000775/1s2.0-S0956713504000775-main.pdf?_tid=2893510a-c4ad-11e394da-00000aacb35f\&acdnat $=1397573554 \_01 f 2 c 2930 b a 5 c 1 e 8$ c4d13914c9762555>. Acesso em 15 abr. 2014. doi: 10.1016/j. foodcont.2004.03.012.

VALADÃO, N.K. Rastreabilidade de micro-organismos patogênicos ao longo da produção de leite pasteurizado: ferramenta potencial para segurança alimentar. 2012. $97 \mathrm{f}$. Dissertação (Mestrado em Ciência da Engenharia de Alimentos) - Departamento de Engenharia de Alimentos - Faculdade de Zootecnia e Engenharia de Alimentos, Universidade de São Paulo, Pirassununga, SP.

VARNAM, A.H.; SUTHERLAND, J.P. Leche y productos lácteos. Zaragoza: Acribia, 1994. p.50-61.

VIOLARIS, Y. et al. Small businesses - big risks: current status and future direction of HACCPin Cyprus. Food Control, v.19, p.439-448, 2008.Disponívelem:<http://ac.els-cdn.com/S0956713507001089/1s2.0-S0956713507001089-main.pdf?_tid=13138ba 0c4ae-11e3-a1ae-00000a a cb362\&acdnat $=1397573947$ e8a3d062ca158be959416635de614c87>. Acesso em 15 abr. 2014. doi: 10.1016/j.foodcont.2007.05.004. 